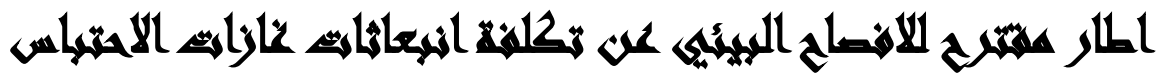

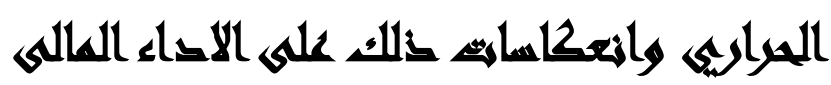

[iv]

جمال سعد خطاب(')- مصطفى خليل(ץ) - مجدى شكري فوزى (r) - رشا محمد عليوه

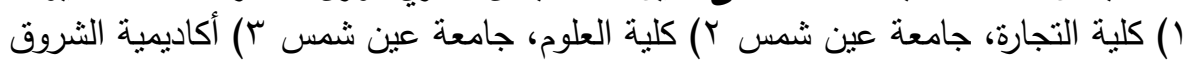

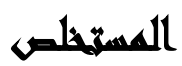

يرهف هذا البحث إلى دراسة العلاقة بين الافصاح البيئي عن تكلفة انبعاثات غازات

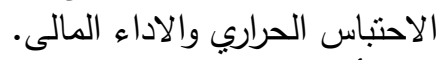

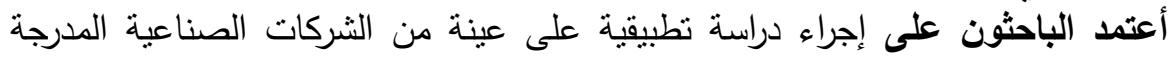

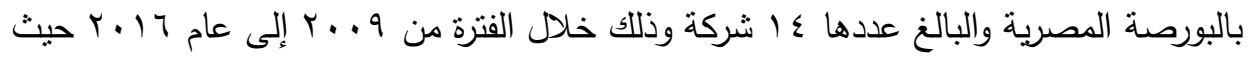
بلغ حجم العينة به ا شركة. قام الباحثون بتجميع البيانات اللازمة لإجراء الإنة الدراسة التطبيقية اعتماداً على عدة مصادر

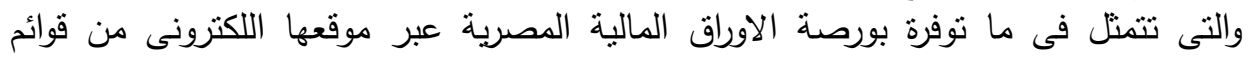

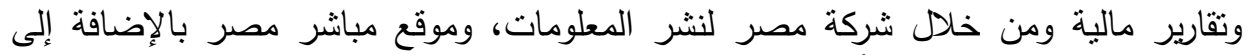

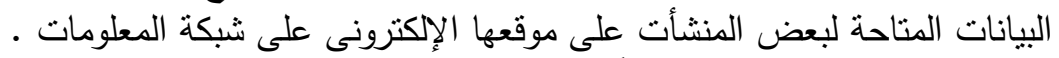

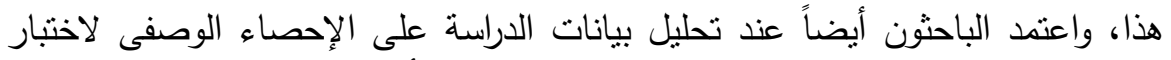

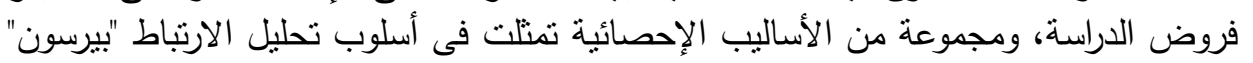
وأسلوب تحليل الانحدار المتعدد.

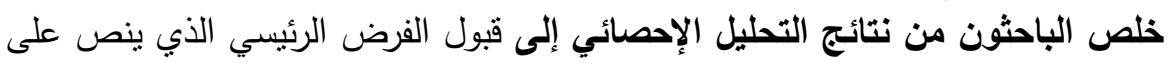

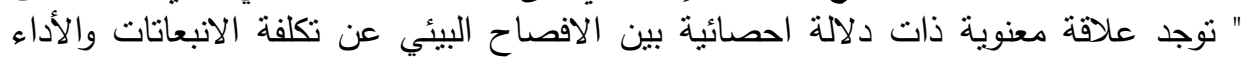
المالى" عند مستوى معنوية (as0.05).

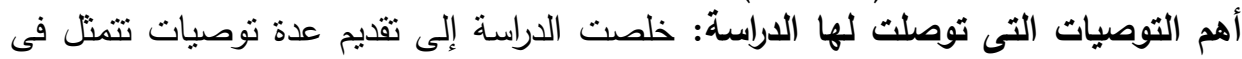

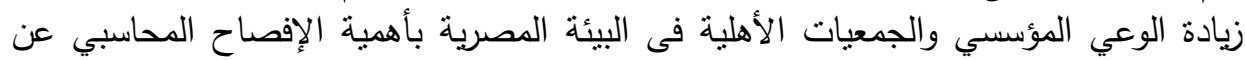

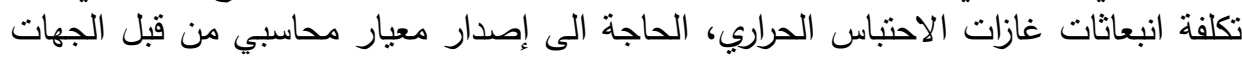

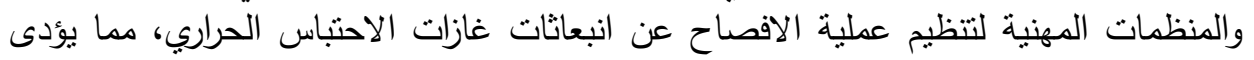

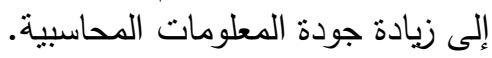
كلمات المفتاحية: الإفصاح البيئي، الاحتباس الحراسية الحري، الأداء المالى 


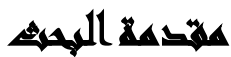

يعتبر التغير المناخى وارتفاع درجه حرارة الارض من أهم وأصعب المشاكل القائمه وأثندها خطراً. وقد أكدت تقارير الهيئه الحكوميه الدوليه المعنيه بتغير الهنير الهناخ (IPCC) Intergovernmental Panel Change

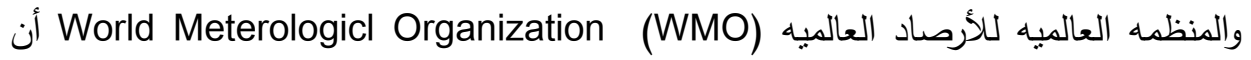
انبعاثات غازات الاحتباس الحراري الناتجه عن المصالح ووسائل واحتراق الوقود الاحفوى

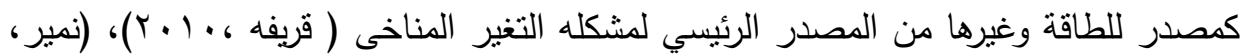

وتعرف ظاهرة تغيير المناخ بأنها اختلال في الظروف المناخية في الظروف المناخية

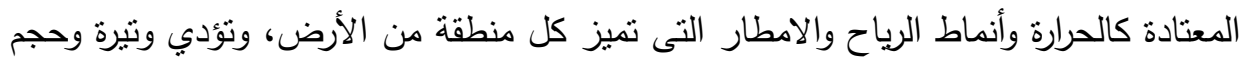

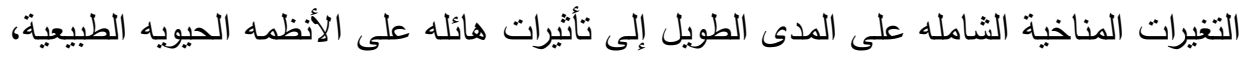

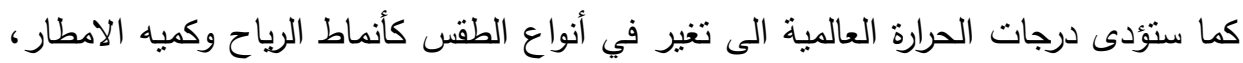
اضافة إلي زيادة احتماليه حدوث الأحداث المناخية المنطرفة، مما يؤدى إلي عواقب بئئيه

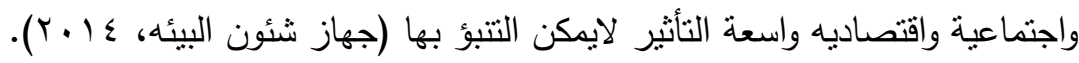

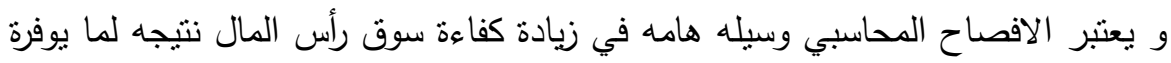

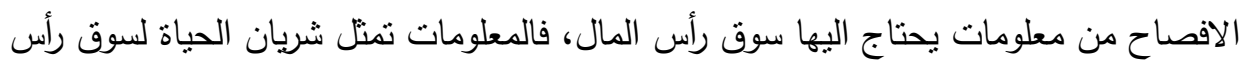
المال، كما أنها تساعد على تخفيض درجه عدم التأكد وتخفيض مشكله عدم تمانل المعلومات. الأمر الذى يؤدى إلي كبر حجم سوق رأس المال متمثنا فى زيادة عدد الأسهم المعروضة لثئه للشراء

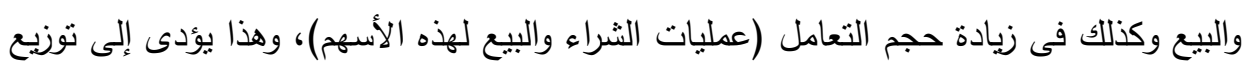
المخاطر بطريقة أفضل بين المشاركين فى سوق رأس المال، وبالتالى التخصيص الامنل للموارد الأقتصاديه، مما يؤدى إلي تحقيق التتمبه الاقتصاديه. إلا أنه فى الفترة الاخيرة كثرت المشاكل والأزمات الماليه نتيجه عدم كفايه المعلومات التهاته المفصح عنها إجبارياً بالإضافة إلى استغلال بعض الأيرة كترت الأطراف وزيادة مشكله عدم تماثل المعلومات بالإضافة إلى استغلال بعض الأطراف للمعلومات الداخليه للشركات بهدف تحقيق

$$
\text { المجلد الأربعون، الجزء الأول، ديسمبر r. V T }
$$


عوائد غير عادية على حساب البعض الأخر ، وكذلك زيادة المضاربه على أسعار الأسهم نتج

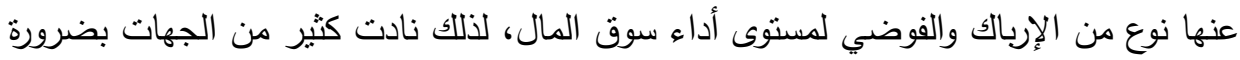
التوسع في الإفصاح المحاسبي.

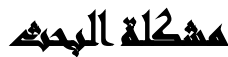

أكد (Flynn, T.2008) الرئيس السابق لمؤسسه KPMG الدوليه أن تغير المناخ لم يعد فى حاجة الى مقدمه ،حيث ينظر اليه الان على نطاق واسع بإعتبارة واحد من أخطر التحديات التى يواجهها العالم بالاضافة الى العواقب التى تتجاوز اثارها على البيئه. كما كما نواجة

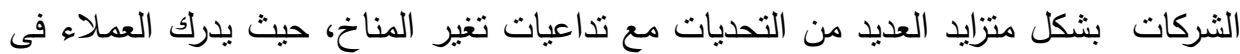
جميع انحاء العالم أن تغير المناخ يشكل مخاطر وفرص على حد سواء، مع الاثار الاستراتيجية والماليه لأعمالهم التجارية.

ويقصد بالافصاح البيئي هـ الاسلوب او الطريقة التى تستطيع المؤسسات من خلالها إعلام المجتمع بأطرافة المختلفة عن نشاطاتها المختلفة ذات المضمون البيئي، وتعد القوائم الماليه أو الثقارير الملحقة بما فيها أداة مناسبه لتحقيق ذلك، كما تعتبر الولايات المتحدة

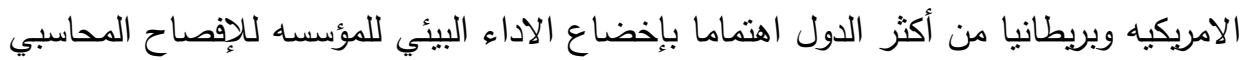

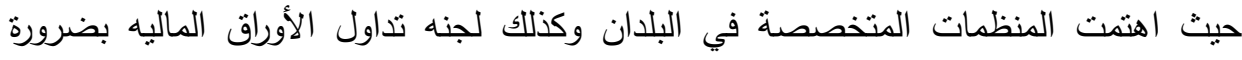
الافصاح عن التأثير البيئي من حيث التكلفة والعائد، كما أوضحت لجنه المعايير بإنجلترا بضرورة الإفصاح عن المعلومات البيئيه والتقرير عن إفصاح المؤسسه عن التكلفة والعوائد

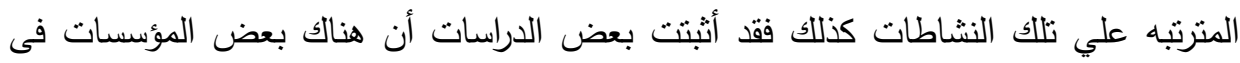

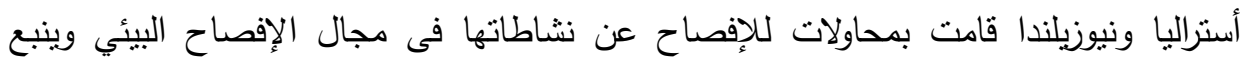

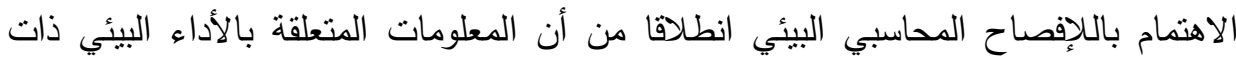

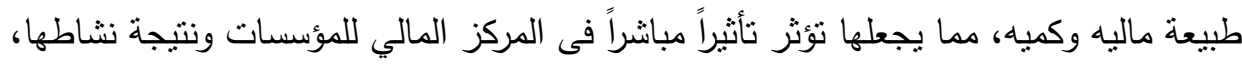
إذ أن القوائم الماليه يجب أن تعكس الألتزامات الفعليه والمحتمله التى تتتج عن عدم التزام المؤسسه بقوانين حمايه البيئة.

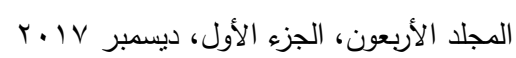


وفي مجال سعي الدول للحفاظ على البيئة وحماية المجتمع فإنه يجب على المنشآت

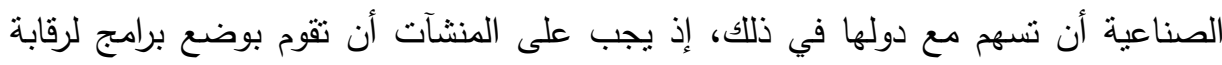

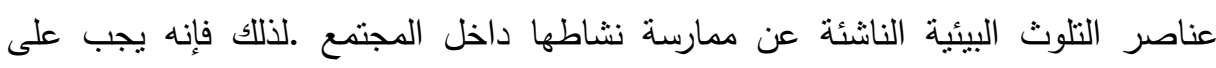
المنشآت أن تفصح عن المعلومات كلّها المرتبطة بشؤون البيئة، وبالعوائد والالتزامات التي لتئي تتحملها نتيجة ممارسة نشاطها المؤثر في لبيئة، خاصةً أن الحاجة للمعلومات البيئية أصبحت

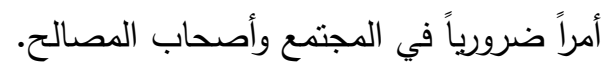
مما أدي الى تزايد الاهتمام بعمليات الافصاح عن الاداء البيئي للشركات ولقد طورت الكثير من الثركات الانظمه البيئيه لديها لتتمكن من إصدار التقارير البيئيه السنويه لتتمكن من التهاه

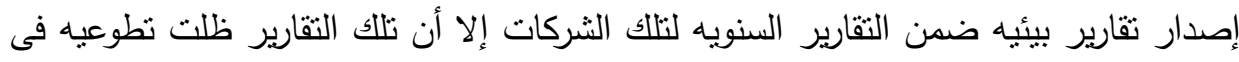
العديد من القوانين بدول العالم المختلفة ومع ذلك هنالك اتجاهاً مضافاً لإتجاة الافصاح عن الإنيه

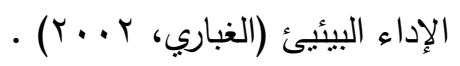

و يرجع السبب فى وجود متل هذا التضارب إلى عدم قيام الهيئات المحاسبيه الدوليه بإصدار معيار أو إرشاد يحدد متطلبات الافصاح اللازم عن الالنزامات البيئيه فى القوائم

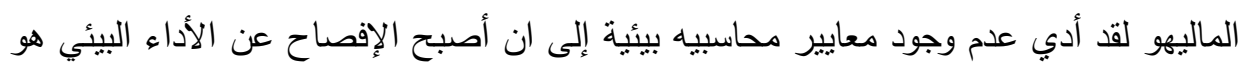

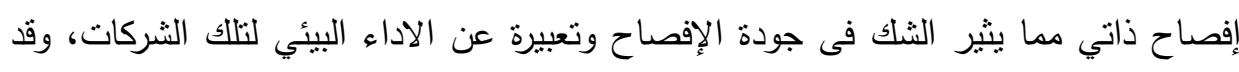

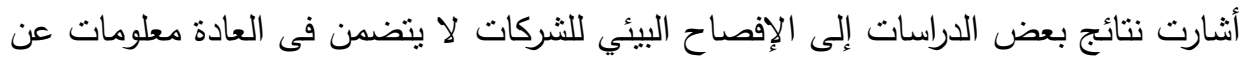

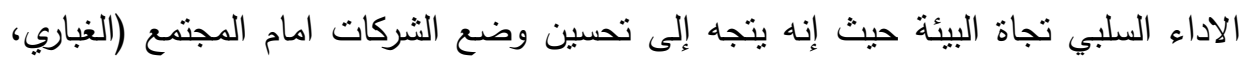

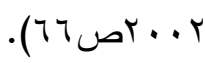
وتتحدد مشكله البحث فى غياب المعايير التى نتتاول الافصاح عن الانبعاثات وقد أدي

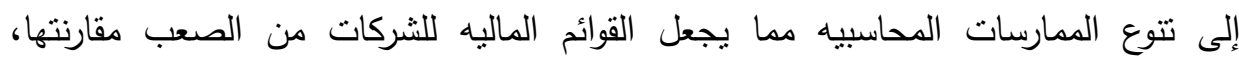
بالاضافة إلى وجود العديد من المخاوف حول الصورة الحقيقية والعادله التى تقدمها القوائم الماليه (خليل، ع ( • ب).

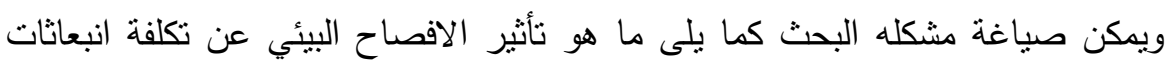
غازات الاحتباس الحراري على الاداء المالي للشركات. 


\section{أهسا هـ المهيه}

يتمثل الهدف الرئيسئ للبحث في وضع اطار للإفصاح البيئي عن تكلفة انبعانات غازات الاحتباس الحراري كأحد الاتجاهات الحديثة للافصاح المحاسبي البيئي ويشتق من هذا لهات الهدف لهن الرئيسى تحديد اثر الافصاح البيئي عن تكلفة انبعاثات الاحتباس الحراري على الاداء المالي.

\section{مروض المهيث}

1-الفرض الأول: وجود علاقة معنوية ذات دلالة احصائية بين مستوى الافصاح البيئي عن تكلفة انبعاثات غازات الاحتباس الحراري والاداء المالى. ץ-الفرض الثاني: وجود علاقة معنوية ذات دلالة احصائية بين التنجيل فى المؤشر المصري لمسئولية الثركات والأداء المالى.

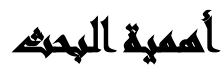

تكمن أهمية هذه الدراسة في الفائدة التي يتم إيضاحها من مبدأ الإفصاح البيئي في الثركات والتي يمكن توضيحها في النقاط الآتية:

1-اهتمام الهيئات والمنظمات المحاسبية والبيئية بإصدار البعض فوضئ من المعايير والنشرات

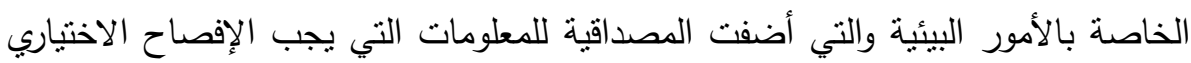
عنه فيما يتعلق بانبعاثات غازات الاحتباس الحراري

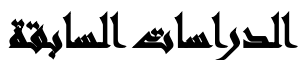

قام الباحثون بمراجعة الأدبيات والبحوث السابقة التى أهتمت بالإفصاح البيئي عن تكلفة

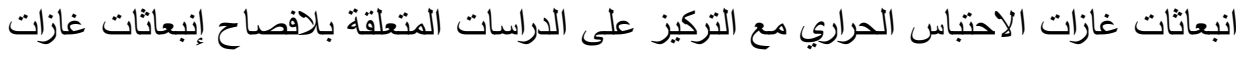
الاحتباس الحراري وذللك للتعرف على اتجاهات هذة الدراسات والمنهجية التى اتبعتها والنتائج التى توصلت إليها. 
1 - دراسة (Prado-Lorenze et al., 2009):

الهذف: هدفت الدراسة إلى تحليل العوامل المختلفة التى تؤثر على الإفصاح عن معلومات

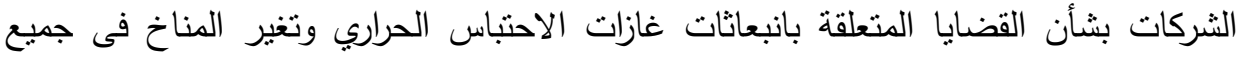
أنحاء العالم. وقد نم إجراء التحليل التجريبي على مرحلتين : تحليل البيانات التى تم الحصول عليها من خلال تحليل المحتوى وتحليل العوامل التى تؤثر على الإفصاح عن انبعاثات غازات الإحتباس

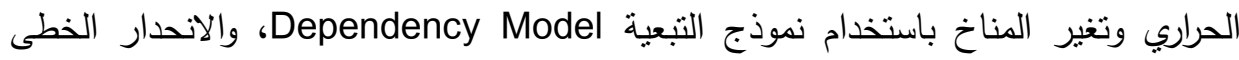
المتعدد وقد نم ادخال العديد من المتغيرات لتمثيل حجم الثركات، والرافعة المالية، والعائد على لئي حقوق المساهمين، ونسبة القيمة السوقية إلى القيمة الدفترية Market to Book Ratio وقد أدرجت المتغيرات الوهمية الأخري : بروتوكول كيوتو، وقطاع النشاط الذى تعمل فية الثركة زادراجها فى مؤشر دواجونز للاستدامة. النتائج: توصلت الدراسة إلى وجود علاقة ايجابية بين الإفصاح عن المعلومات وكل من حجم الثركة ونسبة القيمة السوقية إلى القيمة الدفترية بالإضافة إلى مؤشرات مبادرة التقرير العالمية المقترحة على انبعاثات غازات الاحتباس الحراري، وعلى عكس، نم التوصل إلى وجود علاقة عكسية بين العائد على حقوق المساهمين والإفصاح عن المعلومات. r - دراسه Stanny,2011: الافصاحات الاختياريه عن غازات الاحتباس الحراري وفقا

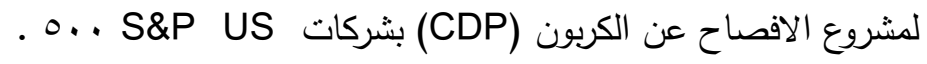

الهاف: اختبار الافصاح الاختباري عن غازات الاحتباس الحراري وفقا لمشروع الافصاح عن

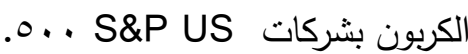
النتائج: توصلت الدراسه الى ان زيادة معدلات الافصاح فى الاتجاهات الحديثة الثلاثة (الاجابه

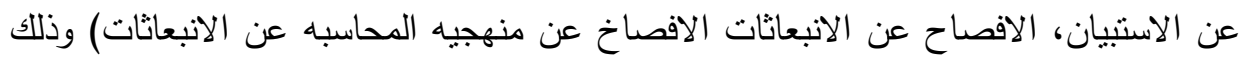

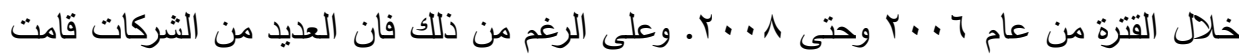

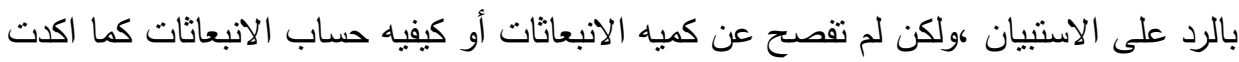
الدراسه إلي ان العامل الاكثر أهيه فى تفسير الافصاحات هو الافصاحات السابقة مما يشير

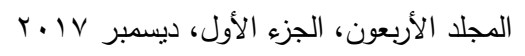


الى الاهيه النسبيه للإفصاحات السابقة مع المقارنه بالعوامل الاقتصادية في تفسير الافصاحات اللاحقة.

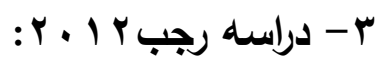

الهدف: يتمثل الهدف الرئيسى للبحث فى دراسه العلاقة بين الافصاح المحاسبي عن انبعاثات غازات الاحتباس الحراري وسلوك اصحاب الدصالح في اتخاذ القرارات.

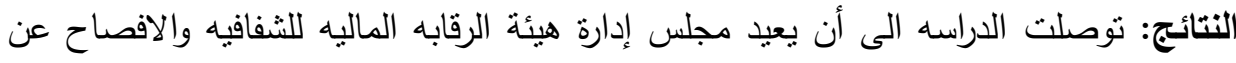
انبعاثات غازات الاحتباس الحراري كمعلومات إضافيه هامه حتى لا يضلل اصحاب المصالح.

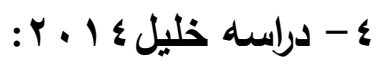

الههف: يتمتل الهدف الرئيسي للبحث في دراسه العلاقة بين خصائص الثركات والافصاح الاختياري لانبعاثات غازات الاحتباس الحراري.

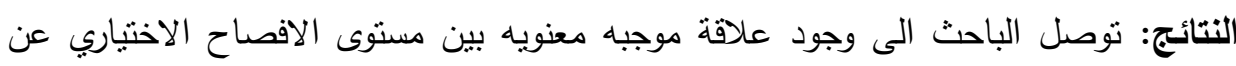

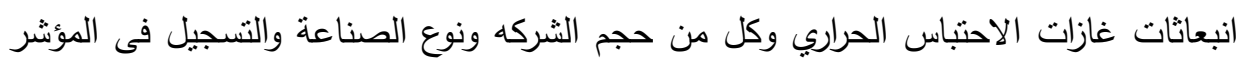
المصري لمسئوليه الشركات.

:Soka, Oshika , 2014 - دراسـ

الهدف: اختبار اثر انبعاثات الكربون والافصاح عن هذه الانبعاثات علي قيمه المنشأه، خاصة

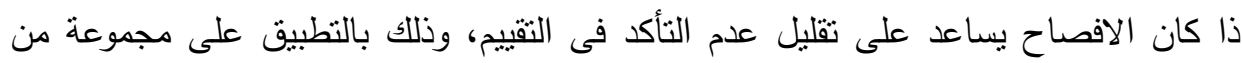
الثركات اليابانيه. النتائج: وتوصلت الدراسه الي أن انبعاثات الكربون للشركه ليها علاقة سالبه مع القيمه السوقيه

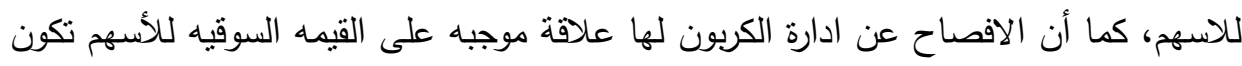
قويه مع وجود كميات كبيرة من انبعانات الكربون.

:Tauringana \& Chithambo, 20144 ع - دراسـ

الهدف: التحقق من تأثثر ارشادات DEFRA, 2009 - إدارة البيئه والثئون الريفيه والغذاء

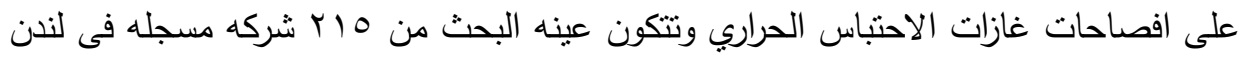

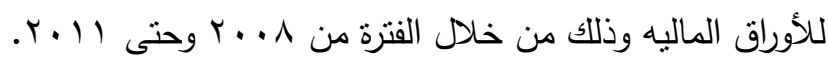

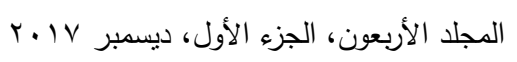




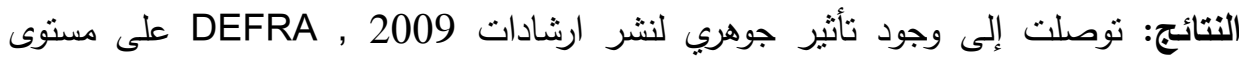

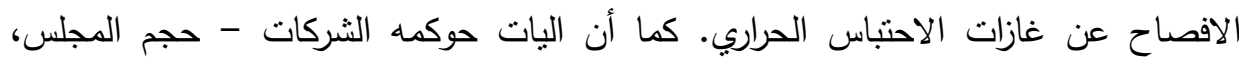

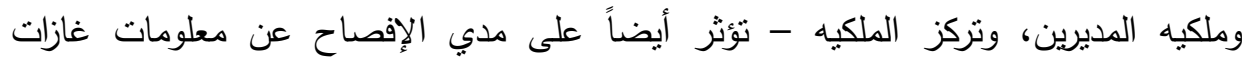

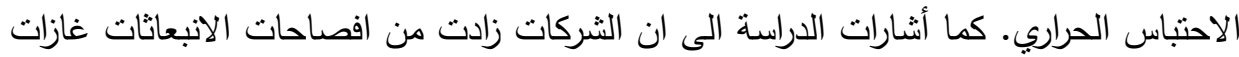

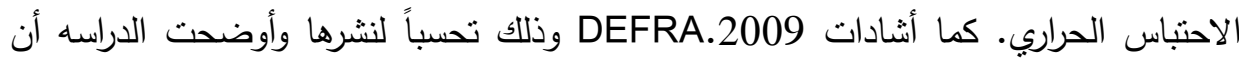
هذة النتائج لها انعكاسات هامه بالنسبه للحكومه، حيث يدل ذللك على أن الارشادات غير وادير الالزاميه من الممكن أن تزيد من الافصاح بقدر ما تفعله المتطلبات الالزاميه.

\section{الإطال النظئيه}

تم تتاول الدراسة السابقة من خلال أربعة محاور على التحو التالى:

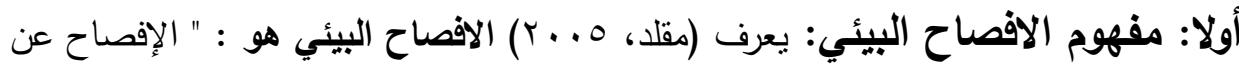
المعلومات البيئية ذات الصلة بأنشطة الثركة إما فى صلب القوائم المالية أو ضمن مرافقاتها، أو فى أي وسيلة عرض أخري للأطراف اصحاب المصالح للمساعدة فى تقييم التداعيات المالية الناشئة عن آثار العمليات الصناعية والمنتجات سواء كان إفصاحاً إلزامياً أو اختيارياً."

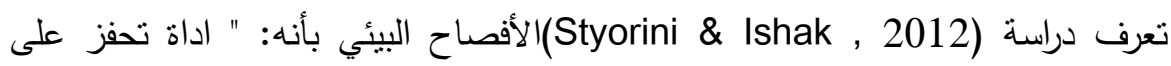

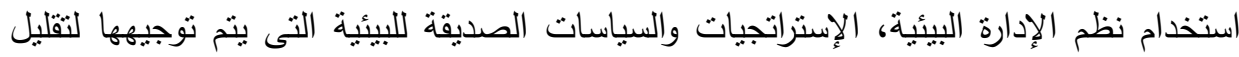

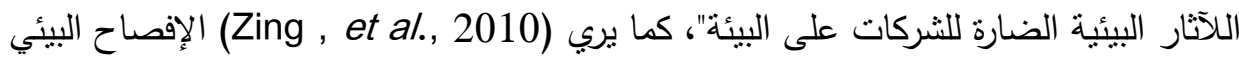

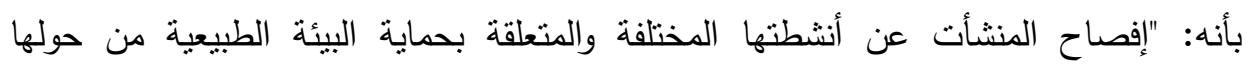
بالإضافة إلى كفاءة استخدام الموارد المتاحة لها". ثانياً: ماهية الاحتباس الحراري: نم اكتشاف الاحتباس الحراري لأول مرة فى القرن التاسع عشر من قبل العلماء مثل (Fourier 1820, Tyndall1863 \& Arrhenius 1896) بدأت ظاهرة الاحتباس الحراري عام • M r فقد وجد أن الغلاف الجوى للأرض كان أكثر نفادية

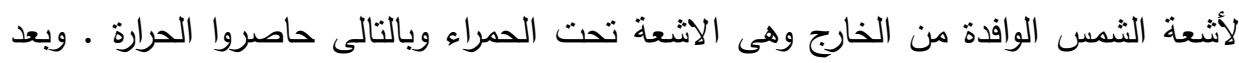

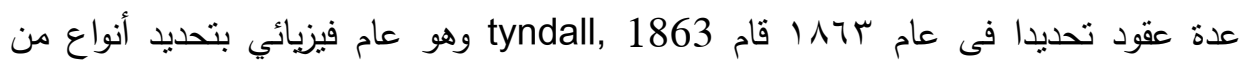

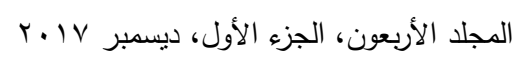


الجزيئات المعروفة باسم (غازات الافيئة)، وعلى رأسها ثانى اكسيد الكربون وبخار الماء الطبيعي

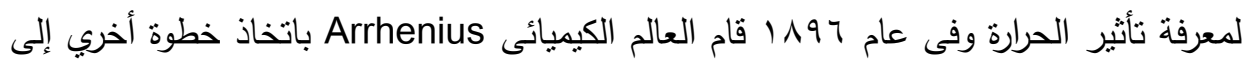
الأحفوري المحترق يؤدى إلى تغيرات كبيرة فى درجات الحرارة السطحية وبالنالى زيادة درجة حرارة الأرض). ولم يستخدم مصطلح الاحتباس الحراري إلا فى الستينيات من القرن العشرين.

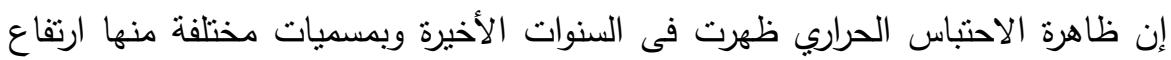
درجة حرارة الارض أو التغيير المناخى (Global Climate Change) أو ظاهرة البيوت أو أو الصوبات الزجاجية (Green House Effect) أو قد تسمى بمشكلة الافيئات باعتبار أن كلمة الدفيئة هى التعريب لكلمة البيت أو الصوبات الزجاجيه وفق السياق المتبع فى بعض الأقطار

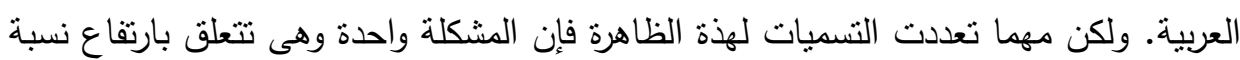

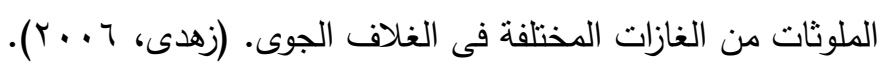
ويري (Travis Wagner) الاحتباس الحراري هو: "زيادة درجة حرارة الأرض نتيجة الفئ احتجاز الغلاف الجوي لكمية أكبر من الطاقة الحرارية المرتدة إلية بالإنعكاس من الأسطح

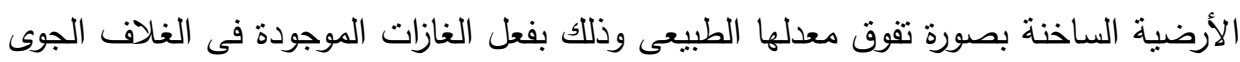

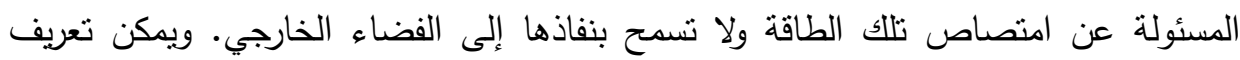

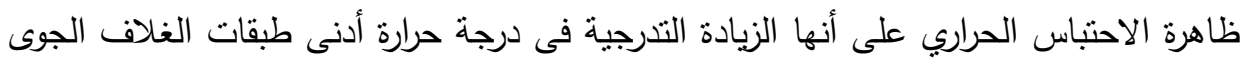

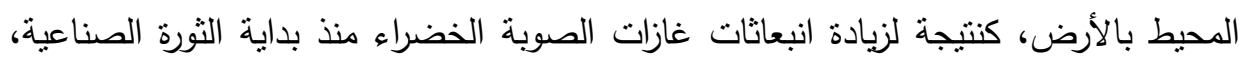

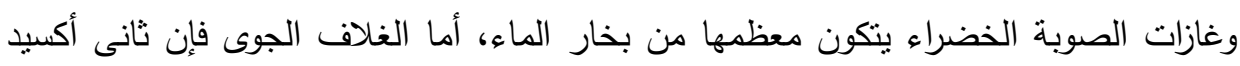

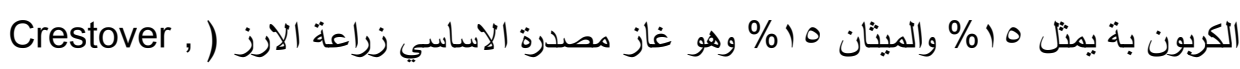

\section{أطر الإفصاح البيئي عن انبعاثات غازات الاحتباس الحراري:}

أولاً: برتوكول غازات الاحتباس الحراري: Greenhouse Gas Protocol : مبادارة برتوكول

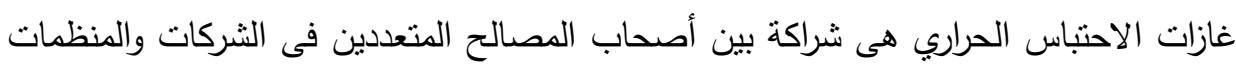
غير الحكومية (NGos)، والحكومات، والاكاديميين وغيرهم والتى عقدها مجلس الأعمال

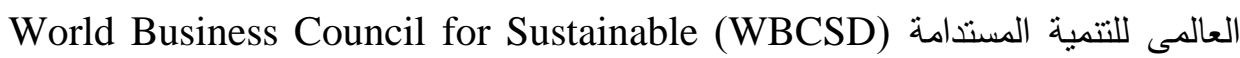

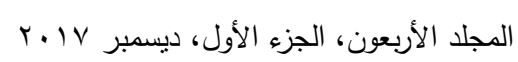


World Resources Institute (WRI) ومعهد الموارد العالمى Development مهمة المبادرة فى تطوير معايير أو برتوكولات للمحاسبة والثقرير عن غازات الاحتباس الحراري

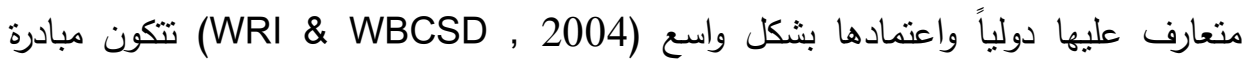
برتوكول غازات الاحتباس الحراري من جزئين منفصلين ولكنهما مرتبطين:

ا لـ معيار المحاسبة والتقرير للشركة. Accounting and reposting

Standard

Project Protocol برتوكول المشروع

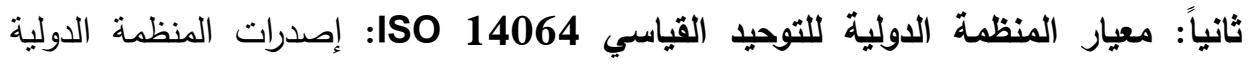

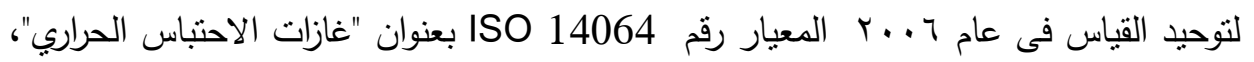
والذى تم تقسيمة إلى ثلاثة أجزاء: الجزع الأول: يتعلق بتوصيف الثركة لمخزونها من تلك الغازات متضمناً بدايه توصيفاً للشركة

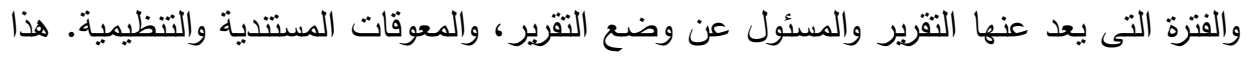
إلى جانب الافصاح عن انبعاثات غازات الاحتباس الحراري المباشر وغير المباشر منفصلة لكل

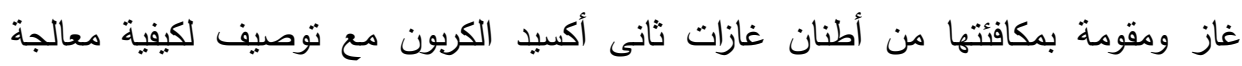
انبعاثات غاز ثانى أكسيد الكربون فى مخزون الانبعاثات.

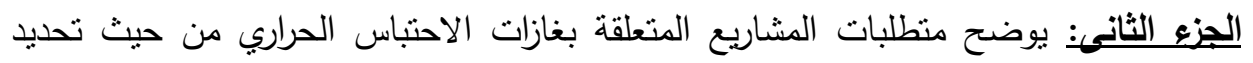
كميات الانبعاثات ومراقبتها وعمل التقارير الخاصة بعمليات خفض الانبعاثات والإرتقاء بعملية

$$
\text { التخلص من الغازات من الغلاف الجوى. }
$$

الجزع الثالث: يوفر المتطلبات والتوجيهات اللازمة لإجراء عمليات التحقق من صحة المعلومات المتعلقة بغازات الاحتباس الحراري ( وينطبق على الثركات التى تجري عمليات التحقق مثل

ثالثاً: الاطار العالمى للافصاح عن المخاطر المناخية(Geres, 2008) : أصدرت مجموعة

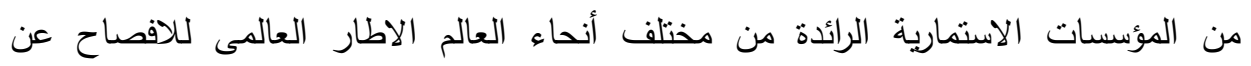

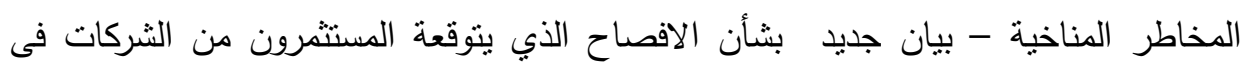

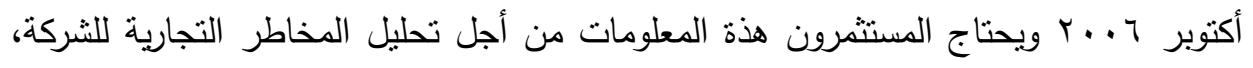
426

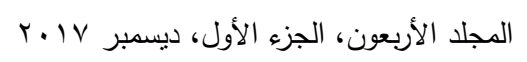


والفرص الناجمة عن تغير المناخ، وكذلك جهود مناخية موحدة حتى يكون من السهل على الثركات توفيرها وكذلك زيادة قدرة المستثرين على تحليل ومقارنة الثركات (CFCRD)

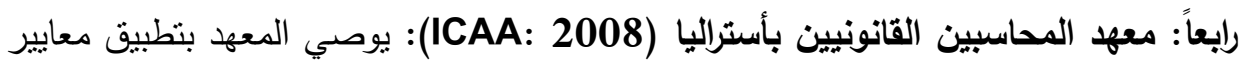
بروتوكول غازات الاحتباس الحراري مع التركيز على الإفصاح عن انبعاثات الكربون وتحديداً

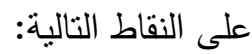
- - الإفصاح عن انبعاثات الكربون وذلك لأغراض مراقبة الدعاوى القانونية من خلال التطورات

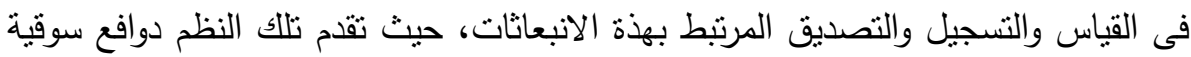
لتخفيض الانبعاثات عندما تكون تكلفة التخفيض فى أقل معدلاتها.

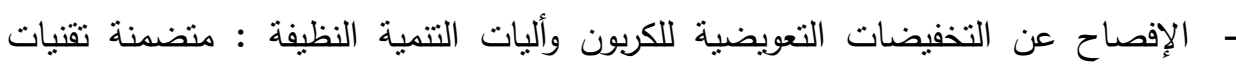
وأساليب التخفيض المطبقة ومنها تقنبات الفحم النظيف، واعادة التشجير وتثنيات التقاط وتخزين الكربون، وكثير من نظم تجارة الكربون الناشئة نسهل التجارة فى مشروعات

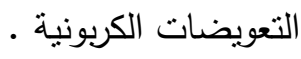

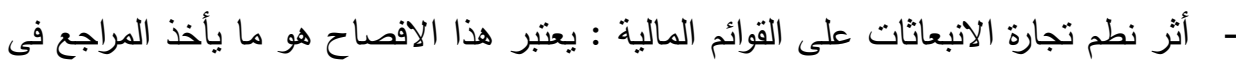

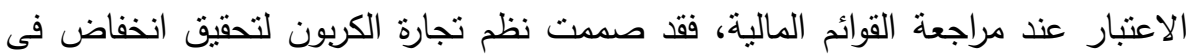
غازات الاحتباس الحراري من خلال استخدام تراخيص الانبعاثات القابلة للتداول.

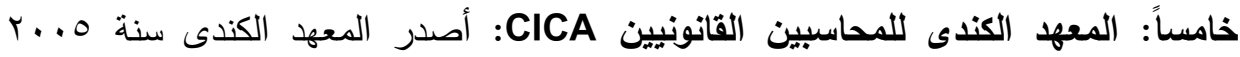
ملخصاً للإفصاح عن الآثار المالية المترتبة على تغير المناخ والقضايا البيئية الأخري فى تقرير

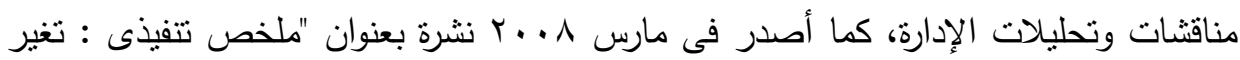

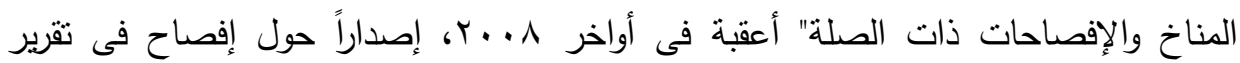
مناقثات وتحليل الإدارة عن تغير المناخ وأخيراً لفت المعهد انتباه المديرين إلى مسؤلياتهر عن الهن مراجعة وإثبات الإفصاحات ذات الصلة بالتغيرات المناخية فى نشرة بعنوان " ملخص الإدرات التغيرات

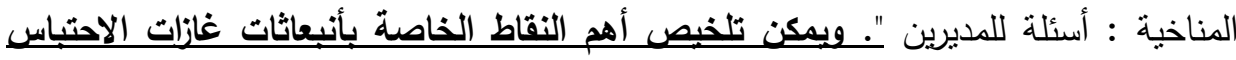

(CICA, 2008) : الحراي فيما يلحئ :

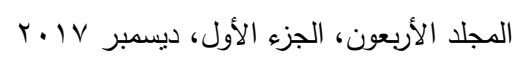


- - تقديم بيانات مباشرة وغير مباشرة عن انبعاثات غازات الاحتباس الحراري: وذلك عن الفترة

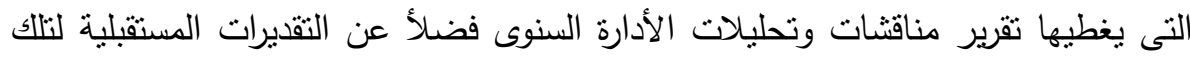
الانبعاثات متضمنة أى أهداف محددة ومعبر عنها فى صورة مطلقة ونسبية، وذلك حتى ونى يمكن تقييم الأداء وجوانب مستقبلية للشركة ككل ولكل قطاع (مركز ربحية). - مقارنة الانبعاثات المستهدفة بالانبعاثات الفعلية مصحوبة بتحليلات وتفسيرات يجب إنباء إجراء

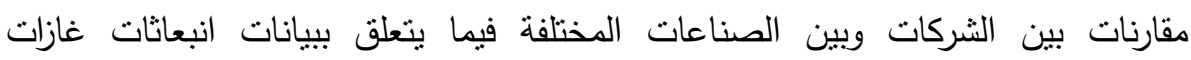
الاحتباس الحراري واتجاهاتها وأهدافها. - - الإفصاح عن أية قيود تحد من اكتمال وامكانية الاعتماد على بيانات الانبعاثات. - الافتراضات والمنهجيات المستخدمة لقياس والإفصاح عن الإنبعاثات ومدى استمرار تطبييقها من فترة لأخري، مشتملة على الأحكام والتقديرات التى تدخل فى تطوير أرقام الأنبعاثات

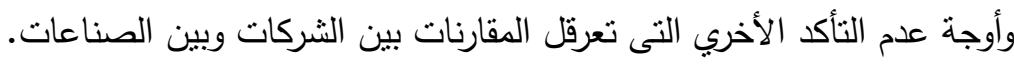

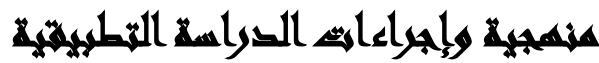

يعتمد منهج الدراسة على الجمع بين المنهجين التاليين:

المنهج الاستنباطى: حيث تم دراسة وتحليل ما تتاولتة الدراسات السابقة التى وردت فى الفكر المحاسبي فيما يتعلق بمتغيرات الدراسة من أجل تحديد مفهوم كل متغير من هذه المتغيرات. المنهج الاستقرائي: حيث يتم وضع اطار مقترح للافصاح البيئي عن انبعاثات الاحتباس

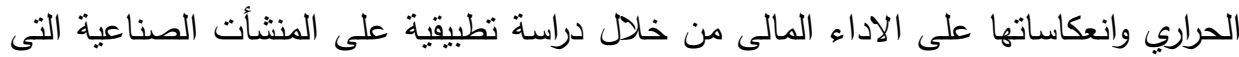
ينتج عنها انبعاثات والمقيدة فى سوق الأوراق المالية المصرية.

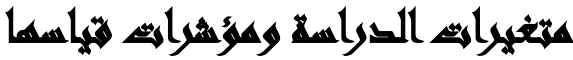

تم تحديد المتغيرات الاساسية فى هذة الدراسة في ضوء طبيعة المشكلة وهما الافصاح البيئي عن تكلفة انبعاثات الاحتباس الحراري كمتغير مستقل، والاداء المالى كمتغير نابع.

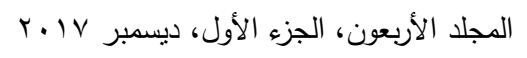


المتغير المستقل: مستوي الافصاح البيئي عن نكلفة انبعاثات غازات الاحتباس الحراري: اعتمد الباحثّن عند قياس مستوي الافصاح البيئي عن تكلفة انبعاثات غازات الاحتباس الحراري على بعض المؤشرات فى الدراسات السابقة وأطر الإفصاح عن انبعاثات غازات الإحتباس الحراري مثل CDSB Protocal، 1-14.64 ISO واصدارات جمعية المحاسبين الكندية والامريكية وانتقاء عناصر الافصاح البيئي التى تلقي اتفاق وقبول الاصدارات المهنية والدراسات السابقة (Tauringana \& Chithambo, 2014). حيث اثتمل هذا المؤشر على ثلاث مجموعات رئيسية تتمثل فى التغير المناخى

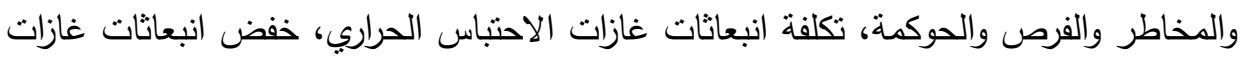

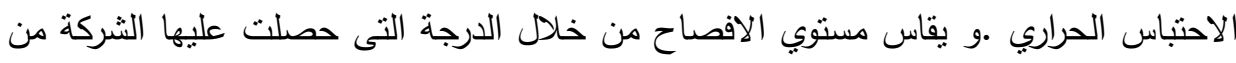
خلال الافصاح عن كل من التغير المناخى : المخاطر والفرص والحوكمة نكلفة انبعاثات غازات الاحتباس الحراري، وخفض انبعاثات غازات الاحتباس الحراري، حيث نأخذ القيمة (1)

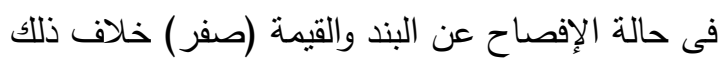

المتغير التابع: الأداء المالى: اعتمد الباحثون قى قياس الأداء المالى للثركات على بعض التص

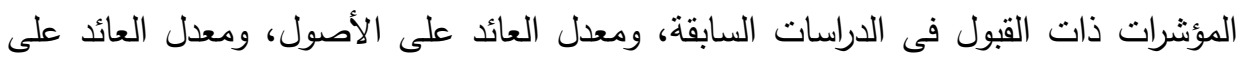
Brine et al, (2007) ; Peters \& Mullen (2009) Karagiorgos ) حقوق الملكية، (2010); Vitezic (2011)) الواردة فى القوائم المالية للثركات موضع الدراسة، حيث إن معدل العائد على الأصول يعتبر

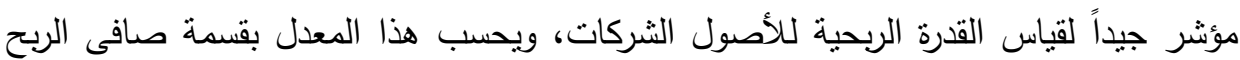

$$
\text { بعد الضرائب على اجمالى الأصول. }
$$$$
\text { المتغيرات الرقابية: }
$$

- كثافة الصناعة Heavy industries : وهو متغير وهمى يأخذ القيمة (1) فى إذا كانت الثركة كثيفة الإنبعاثات، وصفر خلاف ذللك. - التسجيل فى المؤشر المصري لمسئولية الشركات: هو متغير وهمى يأخذ القيمة (1) فى دئ حالة نسجيل الشركة فى المؤشر، و (صفر ) خلاف ذللك.

$$
\text { المجلد الأربعون، الجزء الأول، ديسمبر r.lV }
$$




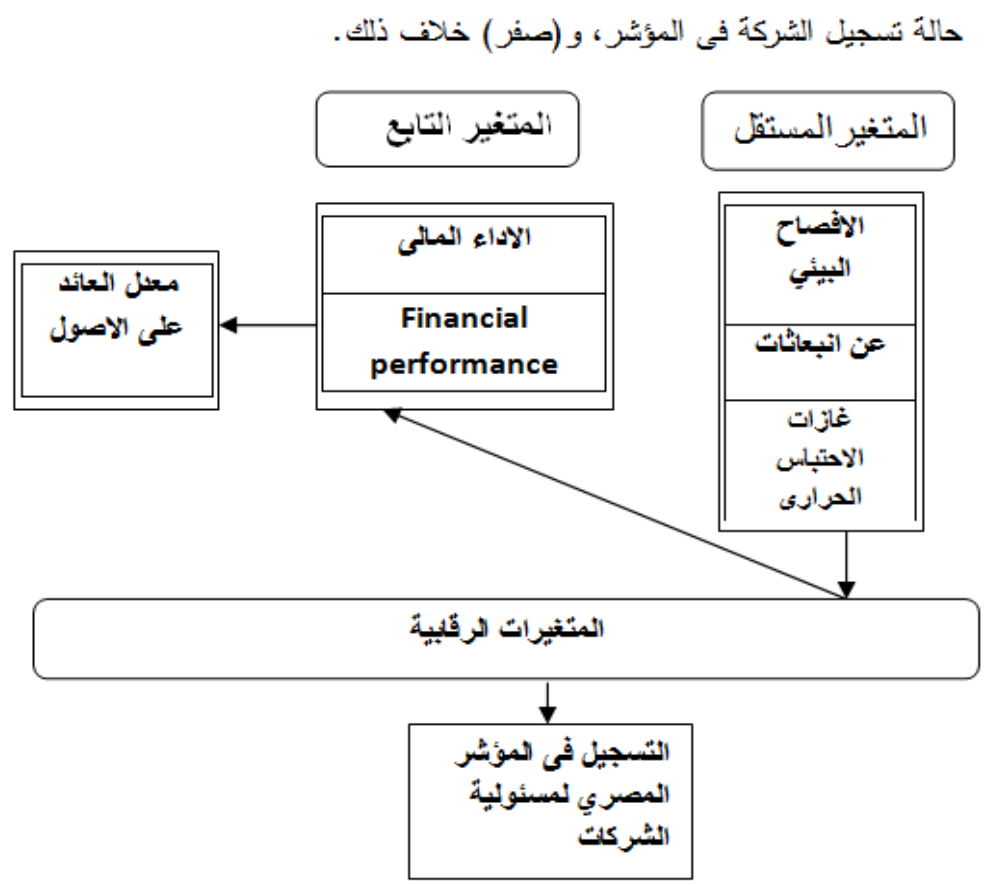

مصادر الحصول على البيانات: اعتد الباحثون فى سبيل الحصول على البيانات الدراسة التطبيقية على عدة مصادر التالية: - ما توفرة بورصة الأوراق المالية المصرية عبر موقعها الالكترونى من قوائم مالية وتقارير

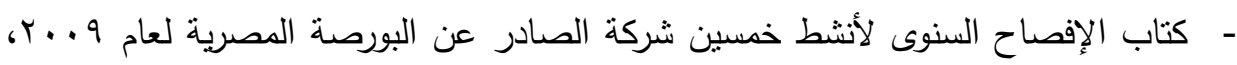

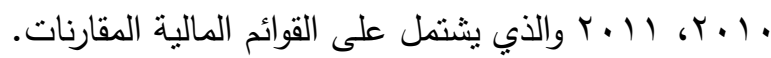
- ـ و ايضا التقارير المالية لبعض الثركات التى نم الحصول عليها من شركة مصر لنشر

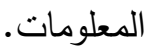
- التقارير المالية التى السنوية التى تقوم الثركات بنشرها اما عبر الموقع الإكترونى للثركة أو موقع البورصة المصرية .ويوضح الثكل رقم (1) متغيرات الدراسة الاساسية. 


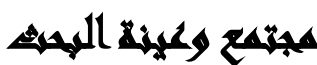

يتكون مجتمع الدراسة من كافة الثركات التى تتداول اسهمها فى سوق الاوراق المالية

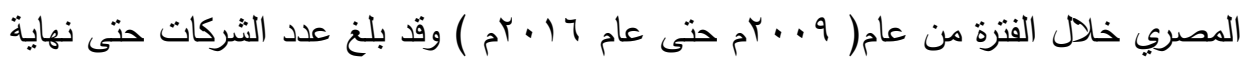

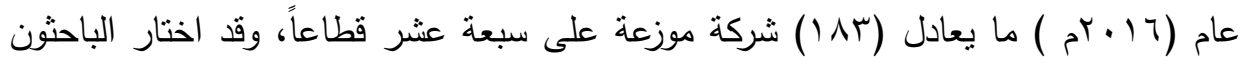
عينة الدراسة وفقاً للشروط التالية: - أن تكون الثركات مقيدة فى بورصة الأوراق المالية المصرية.

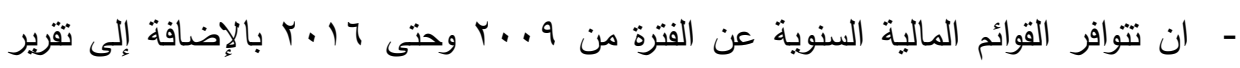
مجلس الادارة والإيضاحات المتممة لإستيفاء البيانات المطلوبة بالنماذج الإحصائية.

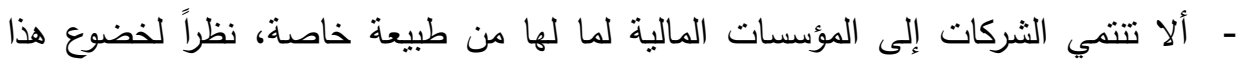
القطاع لمعايير وقوانين خاصة تختلف بطبيعتها عن باقي الثركات.

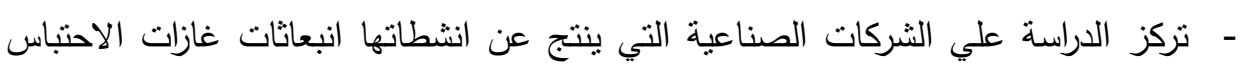
الحراري.

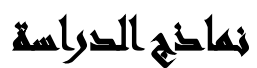

اعتمد الباحثون فى صياغة فروض الدراسة على نموذج الانحدار، وذلك لقياس العلاقة

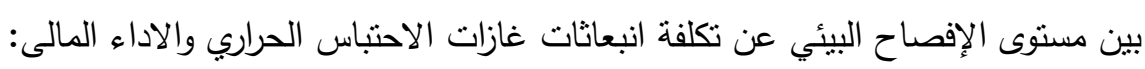

$\mathrm{FP}=\beta 0+\beta 1(\mathrm{GHG}$ Disclosure $)+\beta_{2}(\mathrm{~S} \& \mathrm{P} \mathrm{EGX})+£$

(FP) ق قيمة ثابت الانحدار

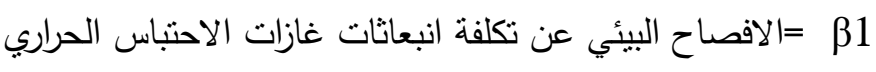
م2 = المتغيرات الرقابية ROA $=\beta 0+\beta 1($ GHG Disclosure)+ $\beta 2($ S\&P EGX Index) (1) : 
ا - نموذج الإنحدار المتعدد لقياس تأثير المتغيرات المستقلة، على معدل العائد

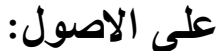

(1) (1) مصفوفة ارتباط بيرسون اهنون جدول رقم(1): مصفوفة ارتباط بيرسون بقياس معنوية العلاقة بين معدل العائد على الاصول والمتغيرات المستقلة

\begin{tabular}{|c|c|c|c|c|}
\hline $\begin{array}{c}\text { S\&P EGS } \\
\text { Indx }\end{array}$ & H-IND & $\begin{array}{c}\text { GHG- } \\
\text { Disclosure }\end{array}$ & ROA & المتغيرات \\
\hline \hline & & & 1.000 & ROA \\
\hline & & 1.000 & ${ }^{*} 0.171$ & GHG-Disclosure \\
\hline 1.000 & ${ }^{*}-.204$ & .103 & ${ }^{*} .159$ & S\&P EGS Index \\
\hline
\end{tabular}

* دالة عند مستوى معنوية أقل من (0. . . ).

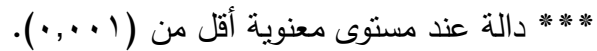

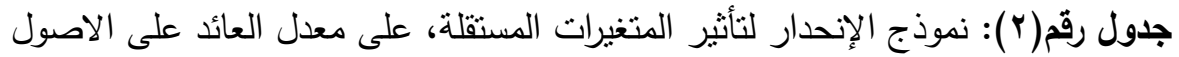

\begin{tabular}{|c|c|c|c|c|}
\hline \multirow[b]{2}{*}{ VIF } & \multicolumn{2}{|c|}{ t. test } & \multirow[b]{2}{*}{ المقدرة المعلمات } & \multirow[b]{2}{*}{ المتغيرات المستقلة } \\
\hline & $\begin{array}{l}\text { Sig } \\
\text { مستوي }\end{array}$ & Value & & \\
\hline 1.16 & *0.0172 & 2.422186 & 0.025074 & GHG-Disclosure \\
\hline 1.31 & 0.1633 & 1.403939 & -0.016851 & SPEGX \\
\hline-- & 0.9460 & 0.067955 & 0.001898 & الجزء الثنابت \\
\hline
\end{tabular}
*دالة عند مستوى معنوية أقل من (1. (., (•).

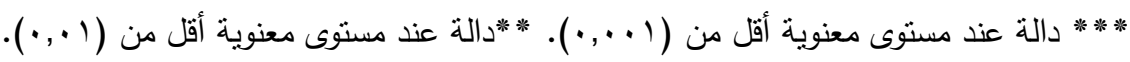


r.test) اختبار معنوية كل متغير مستقل على حدة t-test (خاستخدام اختيار

نجد أن المتغيرات المستقلة ذات المعنوية في نموذج الانحدار الخطي المتعدد هي: الافصاح

البيئي عن تكلفة انبعاثات غازات الاحتباس الحراري، وذلك عند مستوى معنوية أقل من

$$
\cdot(\cdot, \cdot 0)
$$

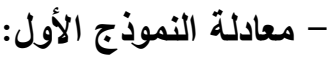

ROA $=0.001898+0.0250739515484 *$ DISCLOSU $+0.0168514199576 *$ SPEGX

وبالتعويض عن قيم المتغيرات المستقلة بالنموذج يمكن الحصول على القيمة المتوقعة

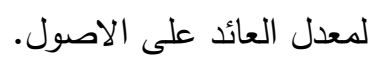

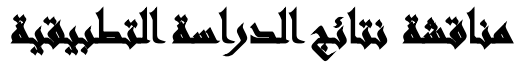

الفرض الاول: " توجد علاقة معنوية بين مستوى الافصاح البيئي عن تكلفة الانبعاثات والاداء

- أظهرت النتائج وجود علاقة ارتباط موجبة معنوية بين مستوى الافصاح البيئي عن تكلفة انبعاثات غازات الاحتباس الحراري ومعدل العائد على الاصول حيث كانت انشارات معات معاملات

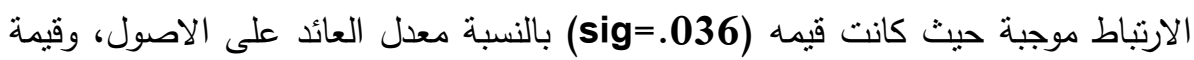

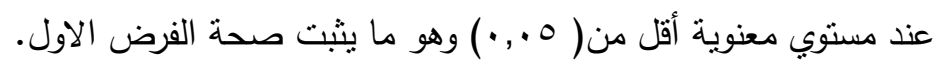
الفرض الثاني: نوجد علاقة معنوية بين التسجيل فى المؤشر المصري لمسئولية الثركات وتكلفة الافصاح عن انبعاثات غازات الاحتباس الحراري.

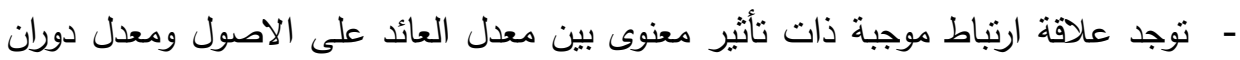

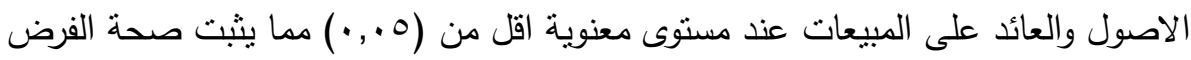




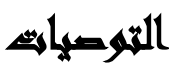

فى ضوء النتائج التى تم التوصل اليها فى الدراسة التطبيقية توصي الباحثة بما يلي: - الحاجة الى إصدار معيار محاسبي من قبل الجهات والمنظمات المهنية لنتظيم عملية الافصاح عن انبعاثات غازات الاحتباس الحراري، مما يؤدى إلى زيادة جودة المعلومات

$$
\text { المحاسبية. }
$$

- - تبنى الهيئة العامة للرقابة المالية أليات من شأنها نلزم الثركات بالإفصاح عن تكلفة انبعاثات غازات الاحتباس الحراري، من خلال تقارير الاستدامة أو التقارير السنوية للشركات بهدف تعزيز قدرة المستثرين على إدراك الفرص الاستثمارية البديلة، ومن ثم اتخاذ القرارات

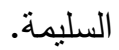

\section{المراليج}

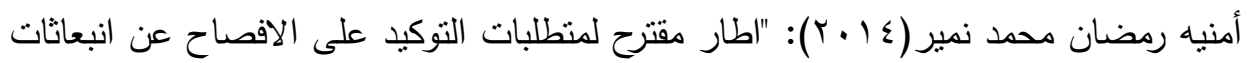

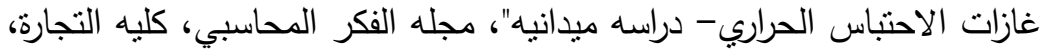

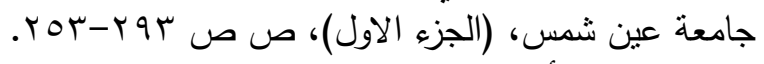

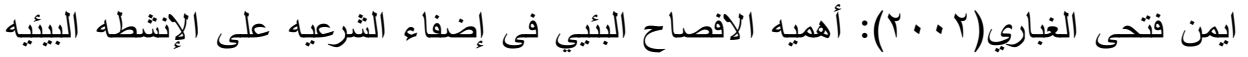

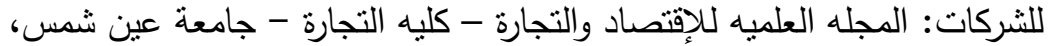

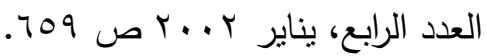

Intergovernmental Panel (IPCC) تقارير الهيئه الحكوميه الدوليه المعنيه بتغير الكناير هناخ

Change

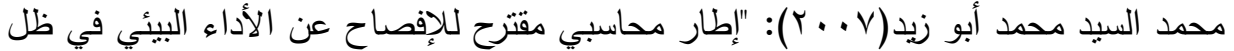

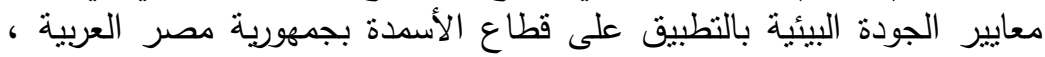

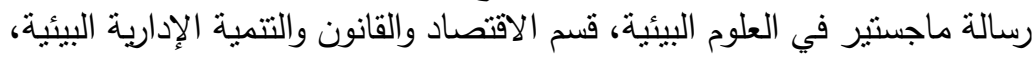

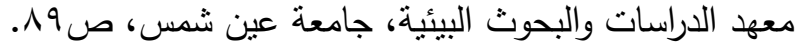

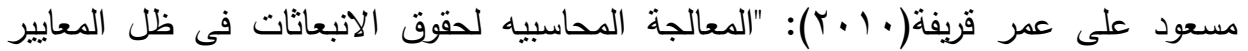

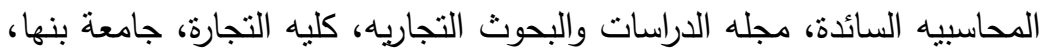

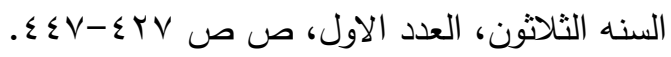




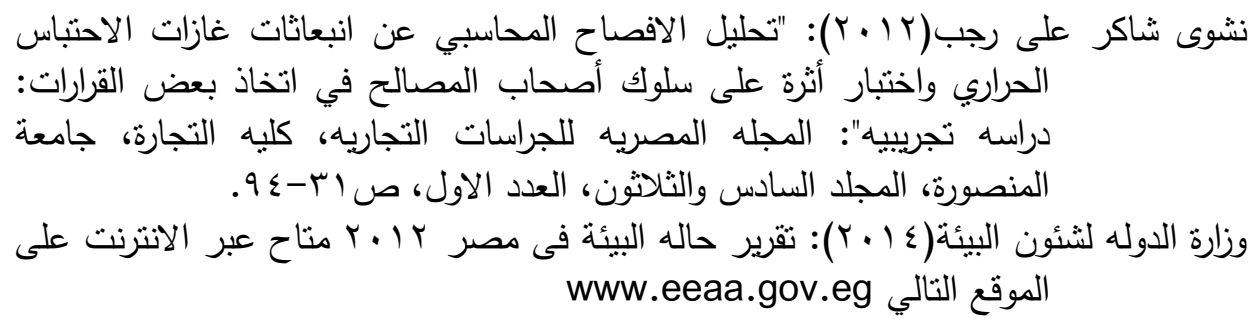

Altintas, N(2013), "Carbon Disclosure Practices in Turkey: A study on ISE listed companies", journal of business and Management ,Vol.13, issue1, (septoct.), pp35-45.

Choi-B. (2013) , "An analysis of Australian Company Carbon Emission Disclosures", Pacific Accounting Review, Vo. 25, No. 1 , PP 58-79.

Prado-Lorenzo, J. (2010), " the role of the board of directors in disseminating relevant information on greenhouse gases", Journal of Business Ethics, 97, pp391-424.

Prado-Lorenzo, (2009), "factors influencing the disclosure of greenhouse gas emissions in companies world wide', Management decision, Vol. 47, No, 7, pp1133-1157. Abstract $\mathrm{id}=1454808$,accessed,20 April,2014.

Rankin, M (2011), " investigation of voluntary corporate green house gas emissions reporting in a market governance systemAustralian Evidence", Accounting , Auditing \&Accountability Journal , Vol.24, No.8, pp 1037-1070.

Saka,C \&Oshika, T., (2014) 'Disclosure effects, Carbon Emission and corporate Value", sustainability accounting Management and policy journal, Vol. 5, No.1,pp.22-45..

Stanny, E, (2011),"Voluntary disclosure of emissions by US firms", working paper, Sonoma state university", pp.1-24available at :http/papers.ssrn.com/s013/.cfm?

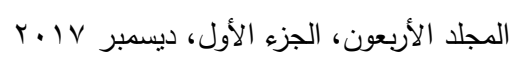




\title{
A PROPOSED FRAMEWORK OF ENVIRONMENTAL DISCLOSURE ABOUT THE GREENHOUSE GAS EMISSIONS AND ITS REFLECTION ON FINANCIAL PERFORMANCE
}

\author{
Khatab, G. S..$^{(1)}$; Khalel, M. ${ }^{(2)}$; Shokry, M. ${ }^{(3)}$ \\ and Elywa, Rasha, $M$
}

1) Faculty of Commerce, Ain Shams University 2) Faculty of Science Ain, Shames University

\begin{abstract}
This research aims to study the relationship between environmental disclosure on the cost of greenhouse gas emissions and financial performance.

The researcher relied on an applied study on a sample of the listed industrial companies in the Egyptian Stock Exchange of 14 companies during the period from 2009 to 2016. The sample size was 183 companies.

The researcher collected the data needed to conduct the applied study based on several sources, which are represented by the Egyptian Stock Exchange through its electronic website from financial lists and reports through Egypt Information Dissemination Company and Mubasher Egypt website, in addition to the data available for some establishments on its website On the Internet

The researcher also relied on the analysis of the study data on descriptive statistics to test the hypotheses of the study, and a set of statistical methods represented in the method of correlation analysis Pearson and the method of multiple regression analysis.

The researcher concluded from the results of the statistical analysis to accept the main assumption that "there is a statistically significant
\end{abstract}

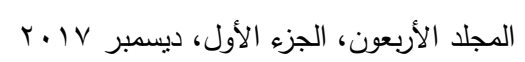


جمال سعد خطاب وآخرون

relationship between the environmental disclosure of the cost of the outputs and the financial performance" at a significant level $(a \leq 0.05)$.

The main recommendations made by the study: The study concluded that several recommendations were made to increase the institutional awareness and the NGOs in the Egyptian environment regarding the importance of accounting disclosure on the cost of greenhouse gas emissions. The need to issue an accounting standard by professional bodies and organizations to regulate the disclosure of gas emissions Global warming, leading to an increase in the quality of accounting information. 